\title{
Coronary physiology assessment in a cardiac transplant patient
}

\author{
L. J. C. van Zandvoort · K. Masdjedi · M. N. Tovar Forero · O. Manintveld · J. Daemen
}

Published online: 8 July 2019

(C) The Author(s) 2019

A 39-year-old male underwent coronary angiography 14 years after cardiac allograft transplantation revealing an intermediate grade stenosis in the mid left anterior descending artery (LAD) for which further physiological assessment was performed (Fig. 1). Subsequent pressure wire-based fraction flow reserve $\left(\mathrm{FFR}_{\mathrm{pw}}\right)$ was 0.87 , suggesting a hemodynamically nonsignificant lesion. However, non-hyperaemic 3-dimensional quantitative coronary angiography-based vessel fractional flow reserve (vFFR) was 0.74 (Fig. 1b). Given the discrepancies, optical coherence tomography was performed showing a fibrofatty plaque with a minimal lumen area (MLA) of $1.70 \mathrm{~mm}^{2}$. The LAD was subsequently treated with a $3.0 \times 15 \mathrm{~mm}$ stent. There has been ongoing debate on the validity of using FFR in denervated hearts due to high rates of microvascular dysfunction and an unreliable hyperaemic response [1]. Angiography based vFFR might be a promising new technology to study the hemodynamic significance of intermediate coronary artery lesions in denervated hearts [2].
Funding The present work was funded by the Erasmus University Medical Center, Rotterdam, the Netherlands

Conflict of interest L.J.C. van Zandvoort, K. Masdjedi, M.N. Tovar Forero, O. Manintveld and J. Daemen declare that they have no competing interests.

Open Access This article is distributed under the terms of the Creative Commons Attribution 4.0 International License (http://creativecommons.org/licenses/by/4.0/), which permits unrestricted use, distribution, and reproduction in any medium, provided you give appropriate credit to the original author(s) and the source, provide a link to the Creative Commons license, and indicate if changes were made.

\section{References}

1. Hirohata A, Nakamura M, Waseda K, Honda Y, Lee DP, Vagelos $\mathrm{RH}$, et al. Changes in coronary anatomy and physiology after heart transplantation. Am J Cardiol 2007;99(11):1603-7.

2. Masdjedi K, van Zandvoort L, Balbi MM, et al. Validation of 3-Dimensional Quantitative Coronary Angiography based software to calculate Fractional Flow Reserve: Fast Assessment of STenosis severity (FAST)-study. EuroIntervention 2019. https://doi.org/10.4244/EIJ-D-19-00466. [Epub ahead of print]. 
Fig. 1 a Coronary angiography, 14 years after allograft cardiac transplant. The LAD shows an angiograpic intermediate stenosis in the midsegment; $\mathrm{Pd} / \mathrm{Pa}$ values in blue and FFR values in red. Optical coherence tomography of the LAD shows a $15 \mathrm{~mm}$ lesion with a minimal lumen area (MLA) of $1.70 \mathrm{~mm}^{2}$ and appropriate landing zones. b Vessel FFR (vFFR) of the LAD. The VFFR is 0.74 , which indicates a significant lesion (threshold $\leq 0.80$ ). $L A D$ left anterior descending coronary artery, $\mathrm{Pd} / \mathrm{Pa}$ distal coronary artery pressure/aortic pressure, FFR fractional flow reserve
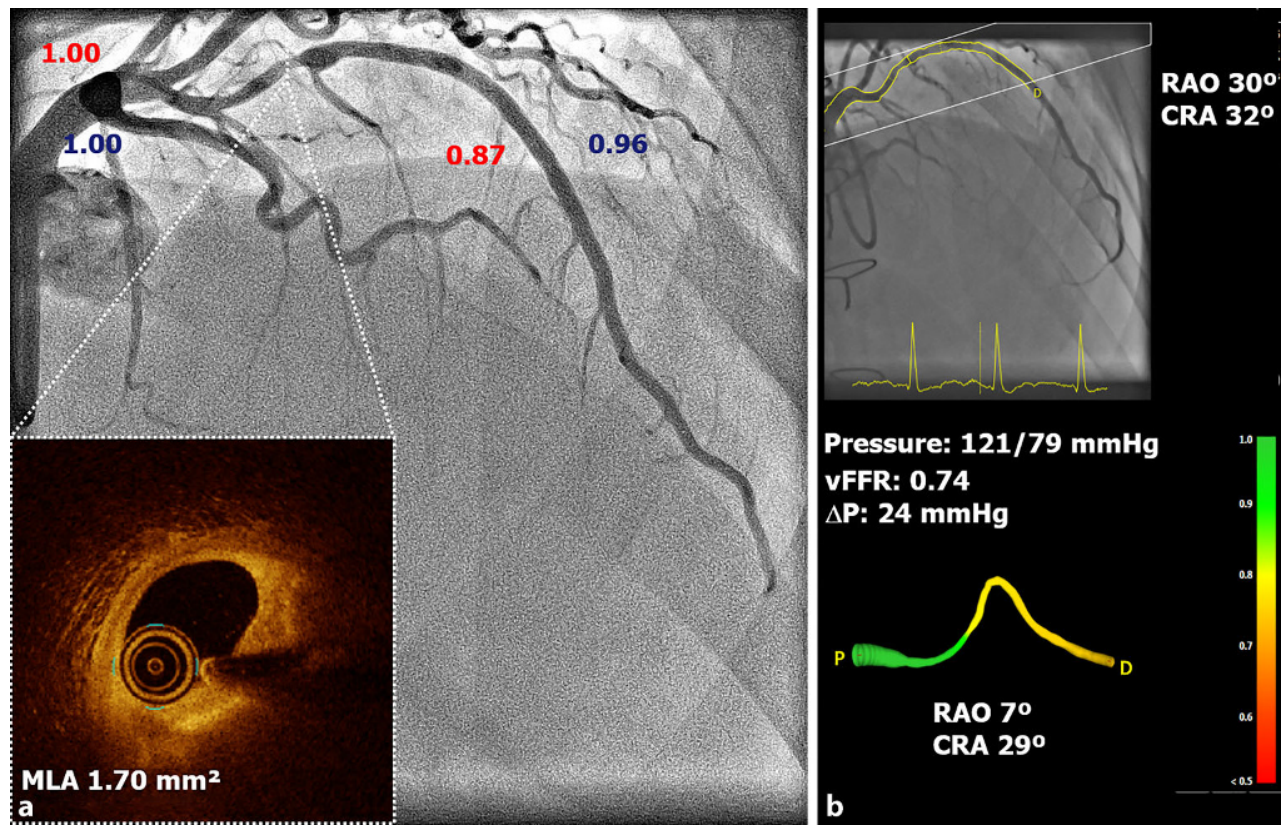